\title{
Evolutionary paths of streptococcal and staphylococcal superantigens
}

\author{
Kayo Okumura ${ }^{1,4}$, Yumi Shimomura', Somay Yamagata Murayama ${ }^{2,5}$, Junji Yagi ${ }^{3}$, Kimiko Ubukata², \\ Teruo Kirikae ${ }^{1}$ and Tohru Miyoshi-Akiyama ${ }^{1 *}$
}

\begin{abstract}
Background: Streptococcus pyogenes (GAS) harbors several superantigens (SAgs) in the prophage region of its genome, although speG and smez are not located in this region. The diversity of SAgs is thought to arise during horizontal transfer, but their evolutionary pathways have not yet been determined. We recently completed sequencing the entire genome of $S$. dysgalactiae subsp. equisimilis (SDSE), the closest relative of GAS. Although speG is the only SAg gene of SDSE, speG was present in only 50\% of clinical SDSE strains and smez in none. In this study, we analyzed the evolutionary paths of streptococcal and staphylococcal SAgs.

Results: We compared the sequences of the 12-60 kb speG regions of nine SDSE strains, five speG ${ }^{+}$and four speG ${ }^{-}$ We found that the synteny of this region was highly conserved, whether or not the speG gene was present. Synteny analyses based on genome-wide comparisons of GAS and SDSE indicated that speG is the direct descendant of a common ancestor of streptococcal SAgs, whereas smez was deleted from SDSE after SDSE and GAS split from a common ancestor. Cumulative nucleotide skew analysis of SDSE genomes suggested that speG was located outside segments of steeper slopes than the stable region in the genome, whereas the region flanking smez was unstable, as expected from the results of GAS. We also detected a previously undescribed staphylococcal SAg gene, selW, and a staphylococcal SAg -like gene, ssl, in the core genomes of all Staphylococcus aureus strains sequenced. Amino acid substitution analyses, based on dN/dS window analysis of the products encoded by speG, selW and ss/ suggested that all three genes have been subjected to strong positive selection. Evolutionary analysis based on the Bayesian Markov chain Monte Carlo method showed that each clade included at least one direct descendant.
\end{abstract}

Conclusions: Our findings reveal a plausible model for the comprehensive evolutionary pathway of streptococcal and staphylococcal SAgs.

Keywords: Superantigen, Streptococcus, Staphylococcus, Fenome comparison, Bayes MCMC

\section{Background}

Bacterial superantigens (SAgs) have been shown to cause the massive activation of host $\mathrm{T}$ cells, strongly influencing immunological disorders. To date, nearly 50 bacterial SAgs and related molecules have been described, primarily from Gram-positive bacteria [1-3]. Streptococcus pyogenes (GAS) is one species of bacteria that harbors SAg genes. Analyses of the entire genomes of 13 GAS isolates have shown that each contains two to seven SAg genes (Additional file 1), almost all located in the prophage

\footnotetext{
* Correspondence: takiyam@ri.ncgm.go.jp

'Department of Infectious Diseases, National Center for Global Health and Medicine, 1-21-1, Shinjuku-ku, Tokyo 162-8655, Japan

Full list of author information is available at the end of the article
}

regions of the genome. In contrast, genes encoding the SAgs speG and smez in GAS strains are not located on these mobile genetic elements, although some are surrounded by transposons. Thus, speG and smez in GAS may have been inherited from an ancestor by horizontal gene transfer. Although speJ in M1 GAS is not located on these mobile genetic elements, speJ is not conserved in the genome sequence of other GAS isolates, except for MGAS6180 (data not shown); in some strains, an SAg similar to speC is called speJ. We recently sequenced the entire genome of Streptococcus dysgalactiae subsp. equisimilis (SDSE) [DDBJ: AP010935] [4], a bacterium that causes life-threatening infectious diseases, including sepsis and streptococcal toxic shock syndrome, similar to GAS

\section{Biomed Central}


[5-7]. Analyses of its sequence showed that SDSE is the closest relative of GAS sequenced to date, with $65 \%$ identity (Additional file 2). Streptococcal bacteria other than GAS, such as $S$. dysgalactiae subsp. dysgalactiae [8] and $S$. equi [9-11], have been reported to harbor more than one gene encoding proteins similar to SAgs. In contrast, targeted microarray analyses of 216 GAS virulence genes including SAgs in 58 SDSE strains isolated from human infections showed that the only SAg gene present in SDSE was speG [12], with about $50 \%$ of SDSE strains not harboring this gene [13-15].

Other representative bacterial SAgs and their related products have been identified in Staphylococcus aureus [2]. At least 20 distinct staphylococcal SAgs have been described, including toxic shock syndrome toxin-1 (TSST-1), staphylococcal enterotoxins (SEs), and staphylococcal superantigen-like proteins (SSL), also called staphylococcal enterotoxin-like proteins (SEls) [1-3]. Almost all staphylococcal SAg genes are located in mobile genetic elements, such as prophages, transposons, plasmids, and pathogenicity islands (PIs). The distribution of these mobile elements among $S$. aureus isolates varies considerably [16]. PIs that harbor the gene encoding TSST-1 can be excised and transduced with high efficiency by a staphylococcal phage [17].

In addition to these staphylococcal SAgs, recent studies have identified staphylococcal superantigen-like proteins (SSLs, also known as SETs), which have structural features similar to those of SAgs but do not possess SAg activity [18]. All of the SSLs described to date are located in mobile genetic elements [2]. Interestingly TSST-1, a functional SAg, shows higher sequence and structural similarity to SSL than to staphylococcal SAgs [18].

Structural analysis of SAgs has suggested that they evolved through the recombination of two smaller $\beta$-strand motifs, similar to the immunoglobulin binding motifs of streptococcal proteins G and L and the oligosaccharide/ oligonucleotide binding family, such as the B subunits of $\mathrm{AB}(5)$ heat-labile enterotoxins, including cholera toxin, pertussis toxin, and verotoxin $[19,20]$. However, the origin and evolutionary pathways of streptococcal and staphylococcal SAgs have not been well described.

To elucidate the origin of streptococcal SAgs based on de novo sequencing of SDSE strains and whole genome sequences, we have analyzed the synteny of the regions surrounding speG and smez in 13 GAS and 9 SDSE genomes. We also analyzed the genomic structures of all $S$. aureus strains for which whole genome data are available. We detected a previously undescribed gene that encodes a SEA-like protein (designated selW [21]) and genes encoding SSL-like proteins, all of which are conserved in all $S$. aureus strains sequenced to date and are located in the core chromosome, not in any mobile elements. These findings, in addition to amino acid substitution analyses based on window analysis, cumulative TA-skew analysis and evolutionary analysis according to the Bayesian Markov chain Monte Carlo method, which allows the evolutionary path of SAg to be determined in chronological order, we were able to trace the origin and molecular evolution of streptococcal and staphylococcal SAgs.

\section{Results and discussion}

\section{Comparison of sequences of speG regions in the GAS and} SDSE genomes

The complete sequencing of the entire SDSE genome enabled us to gain insight into the origin of streptococcal SAgs. To elucidate the evolutionary pathways of streptococcal SAgs, it was first necessary to distinguish orthologous from paralogous SAgs in streptococcal genomes. This can be accomplished by syntenic mapping of the genes in regions of interest. Since speG and smez are conserved in almost all GAS genomes, but show low sequence similarities at the nucleotide level (35\%), they are likely distinct direct descendants of ancestral streptococcal SAgs. Inasmuch as some of the regions surrounding speG in the GAS genomes harbor genes encoding putative transposases, which mediate the mobilization of the surrounding genes (Figures 1 and 2), and since speG and smez have low GC contents compared with their surrounding regions (data not shown), we cannot exclude the possibility that speG and smez are also paralogous genes. By analyzing the entire genome of the SDSE strain GGS_124 [DDBJ: AP010935] [4], we could compare its genome with those of other bacteria. We found that this SDSE genome was $65 \%$ identical in sequence to that of the GAS genome (Additional file 2), the highest to date among sequenced bacterial genomes. This finding strongly suggested that SDSE and GAS evolved from a common ancestor, despite SDSE harboring only the putative SAg gene speG. We therefore analyzed the syntenic homology of the speG regions of GGS_124 and GAS strains. We found that, at the amino acid level, speG in GGS_124 was $79 \%$ to $83 \%$ similar to the speG regions of GAS strains.

To exclude the possibility that speG was acquired from a streptococcal phage, we compared the $50 \mathrm{~kb}$ sequences surrounding speG, a size sufficient to detect sequences derived from prophages. Synteny maps of the respective speG regions were essentially conserved in GAS strains and GGS_124 (Figure 1A), except for MGAS10750, which did not harbor the speG sequence present in the corresponding speG regions of GAS and GGS_124. We found that the speG region of each GAS genome contains two to ten genes, which encode factors similar to mobile elements and phage-related genes, such as transposase, IS 


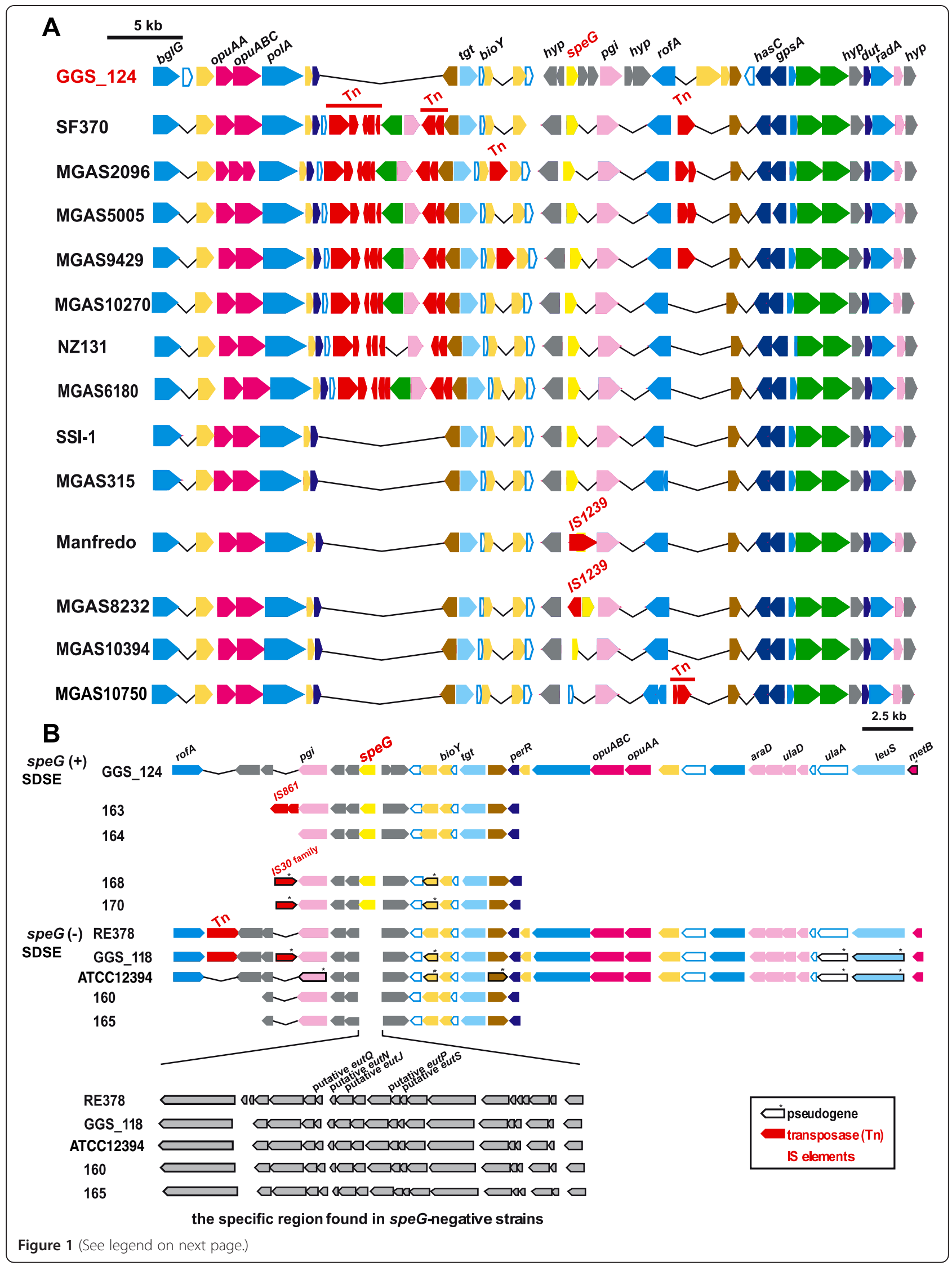


(See figure on previous page.)

Figure 1 Synteny mapping of speG regions in SDSE and GAS genomes. (A) Genome context of speG and the 50 kb surrounding regions of the GGS_124 and 13 GAS strains. Each position (bp) on each genome is shown in Additional file 10. Some sequences encoding small peptides (20 to 30 amino acid residues) were annotated as having unknown functions or as hypothetical proteins and were omitted from this figure, because their assignments depended on the annotator. Transposase and IS elements are shown in red. hyp (in grey) indicates sequences encoding 'hypothetical proteins'. Genes of the speG region of GGS_124 were inversely aligned. Pseudogenes are marked with asterisks. (B) Genome context of speG or the corresponding region in $\operatorname{speG}(+)$ and speG(-) SDSE strains. All information on the strains used in this study is shown in Additional file 1.

and co-activator of prophage gene expression. In contrast, these mobile elements could not be detected in the corresponding speG region of GGS_124 (Figure 1A). The synteny of the regions surrounding the speG gene was highly conserved in eight GAS genomes (i.e. SF370, MGAS5005, MGAS2096, MGAS9429, MGAS10270, SSI-1, NZ131, and MGAS6180), each of which contains seven to eight transposase- and phage-related genes. These regions were $94 \%$ to $100 \%$ identical with each other. In the Manfredo genome, we found that IS1239, which is widely distributed in various isolates of GAS [22], had been inserted into the speG coding sequence, resulting in speG being a pseudogene in this strain (Figure 1A). In the MGAS8232 genome, IS1239 flanked speG.

Although a previous study suggested that speG transferred from SDSE to GAS [23], our results clearly indicate that the synteny surrounding speG in the GAS and GGS_124 genomes has been essentially conserved and that modifications of this context, by insertion of mobile elements, occurred only in GAS strains. These results strongly suggest that speG in GAS and SDSE is an orthologous, not a xenologous, gene, the latter defined as a gene displaced by horizontal transfer from another lineage [24]. Moreover, speG in GAS and SDSE is a descendant of an ancestral streptococcal SAg and has been conserved in evolution.

We next performed amino acid substitution analysis, based on window analysis, to estimate the number of nonsynonymous $(\mathrm{dN})$ and synonymous $(\mathrm{dS})$ substitutions per site for speG and pgi, a housekeeping gene that encodes glucose-6-phosphate isomerase, in GAS and SDSE strains. The ratio of non-synonymous to synonymous substitution rate $(\mathrm{dN} / \mathrm{dS})$ can be used to determine patterns of molecular evolution, with $\mathrm{dN} / \mathrm{dS}>1$ indicating positive selection, $\mathrm{dN} / \mathrm{dS}=1$ indicating neutral selection, and $\mathrm{dN} / \mathrm{d} S$ $<1$ indicating purifying selection. A comparison of speG in GAS and SDSE genomes revealed five peaks with $\mathrm{dN} /$ $\mathrm{dS}>1$ (Figure 3A), suggesting positive selection in these five regions. Crystal structures of $\mathrm{SpeC}$, the SAg protein structurally most similar to SpeG, suggested that several highly conserved domains, including Lys88-Leu97, Gln154-Thr167, and Asp188-Phe197, are important for protein function [25]. Interestingly, the $\mathrm{dN} / \mathrm{dS}$ ratios in these conserved regions were low, suggesting that positive selection pressure in speG operates to conserve a function other than superantigenic activity, because none of the SDSE clinical strains expressed SpeG proteins [13-15]. For comparison, we performed window analysis on the pgi gene, but no $\mathrm{dN} / \mathrm{dS}$ peak above one was observed (Figure 3B), despite this gene being adjacent to speG.

\section{Comparison of the speG region among SDSE strains}

Although the synteny of speG regions of GAS strains and GGS_124 has been highly conserved, about 50\% of SDSE strains do not harbor speG [13-15]. We therefore selected nine SDSE isolates, five with (GGS_124, 163, 164, 168 and 170) and four without (RE378, SDSE_118, 160, and 165) speG (Additional file 1). Following direct genome sequencing (GGS_124 and RE378) or PCR amplification using spe $G$ specific primers (Additional file 3), we compared the sequences of these nine strains. Each of these isolates harbored a different emm type (Additional file 1), widely used to type GAS and SDSE strains [26]. We also included the full sequence of the ATCC 12394 genome [GenBank: CP002215], an SDSE that does not harbor speG [27].

When we analyzed the genetic structures surrounding speG (12 to $60 \mathrm{~kb}$ ) in these SDSE isolates (Figure 1B), we found that, in general, these structures were highly conserved, especially in the $12 \mathrm{~kb}$ regions between $p g i$ (pink) and perR (blue), but that speG itself and its corresponding regions were not. Outside these $12 \mathrm{~kb}$ regions, we found that most of these strains contained 1 or 2 coding sequences similar to transposase or IS elements, including several that appeared to be common to the sequenced GAS genomes.

Remarkably, all four speG-negative strains (e.g. RE378, SDSE_118, 160, and 165) showed the insertion of an approximately $20 \mathrm{~kb}$ fragment between the hypothetical protein gene (locus_tag: SDEG_1990) and the gene similar to peptidoglycan endo-beta- $\mathrm{N}$-acetylglucosaminidase (locus_tag: SDEG_1992) present in the GGS_124 genome, replacing speG (locus_tag: SDEG_1991) at the exact same site. These $20 \mathrm{~kb}$ fragments were composed of 19 or 22 coding sequences, which were similar to genes derived from evolutionally distant species such as Clostridium botulinum and C. tetani. However, the arrangements of these genes did not exactly match those of the clostridial genomes (data not shown), with most coding sequences sharing $<60 \%$ similarity (e.g. Additional file 4). In contrast, genetic structures other than these $20 \mathrm{~kb}$ 


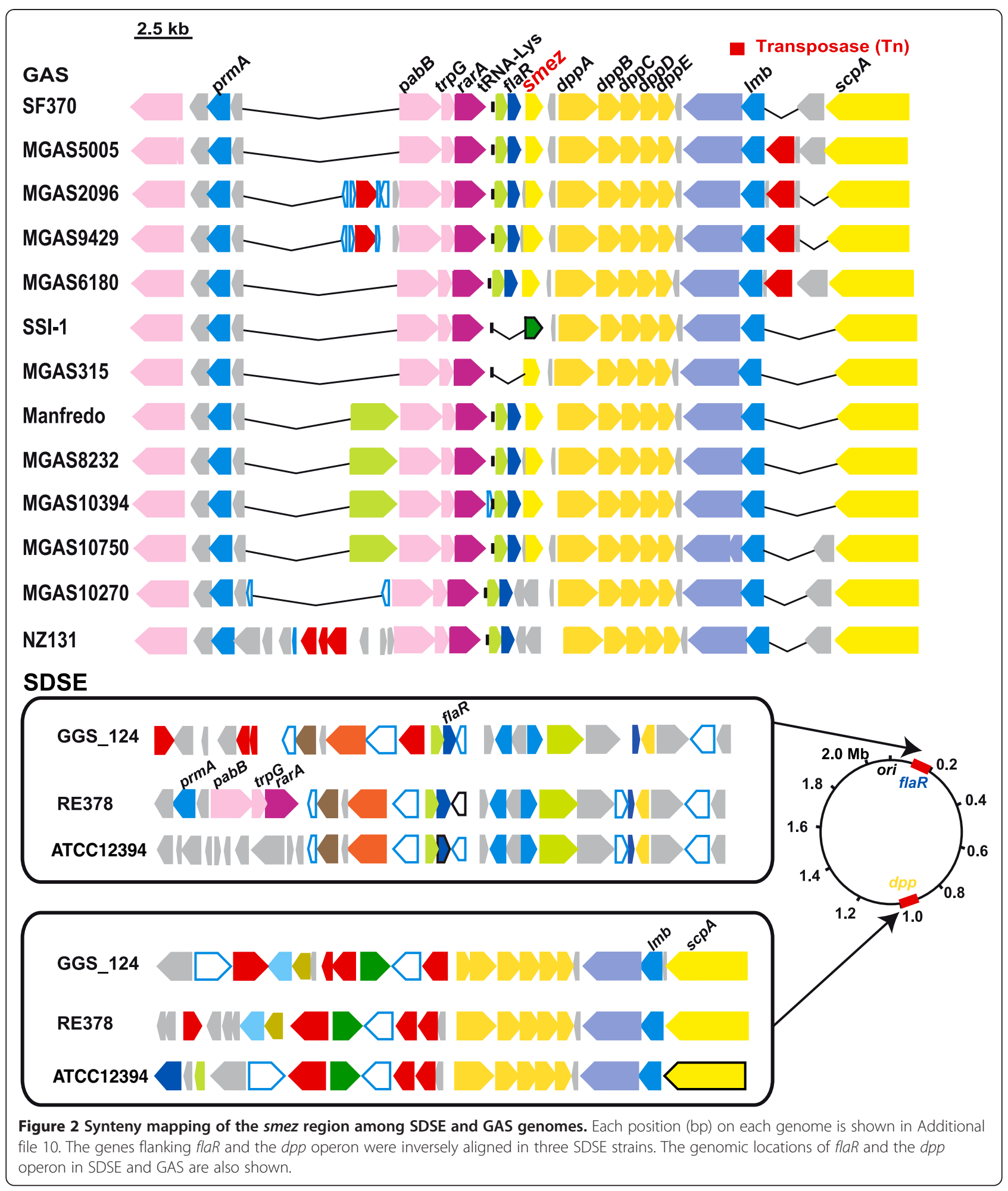

fragments were highly conserved among the speG-positive and -negative strains (Figure 1B). These findings indicated that synteny had been conserved in the regions surrounding speG, or the inserted $20 \mathrm{~kb}$ fragments, of these SDSE strains.
Analysis of the expression of speG region genes

Our finding, that the genomic structures surrounding speG in GAS and SDSE strains are highly conserved, even in speG-negative SDSE strains, suggested that speG in some ancestral SDSE strain had been replaced by a 

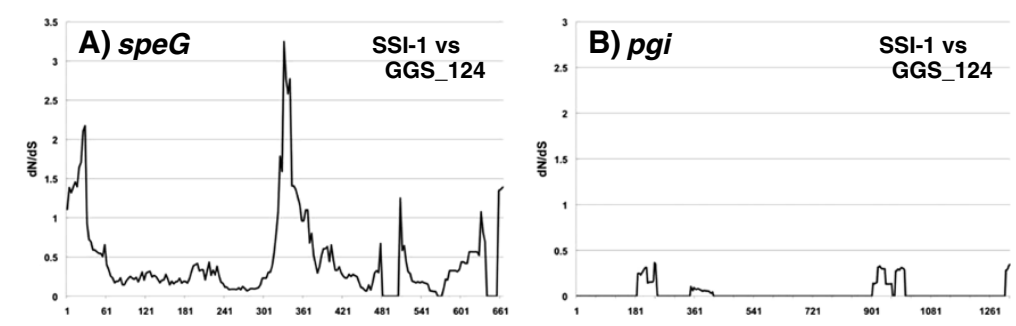

Figure 3 Window analysis calculating $\mathrm{dN} / \mathrm{dS}$ between GAS and SDSE. $\mathrm{dN} / \mathrm{dS}$ was calculated as the ratio of nonsynonymous (dN) to synonymous (dS) substitution rates of gene pairs in speG (A) and pgi (B) from GAS and SDSE genomes. The figure shows only the results of comparisons between SSI-1 (GAS) and GGS_124 (SDSE). The X-axis represents nucleotide position.

$20 \mathrm{~kb}$ fragment soon after the speciation of GAS and SDSE. These types of replacement may take place more frequently among non-functional than among functional genes. To test this hypothesis, we analyzed the expression of genes surrounding speG by RT-PCR, using total RNA isolated from two strains, GGS_124 (SDSE) and MGAS6180 (GAS). In contrast to MGAS6180, which expressed all of the genes analyzed in this study (Figure 4A), GGS_124 expressed all except speG, a difference that may be due to differences in nucleotide sequences at the speG promoter site. When we looked for putative promoter sites $-35 \mathrm{bp}$ and $-10 \mathrm{bp}$ upstream of speG, we found two (Figure 4B), both of which displayed a single nucleotide difference between GGS_124 and MGAS6180. We also identified one mutation each in the second promoter candidate of GAS strains Manfredo, SF370, and MGAS5005, although these mutations did not affect the predicted promoter score (data not shown). In

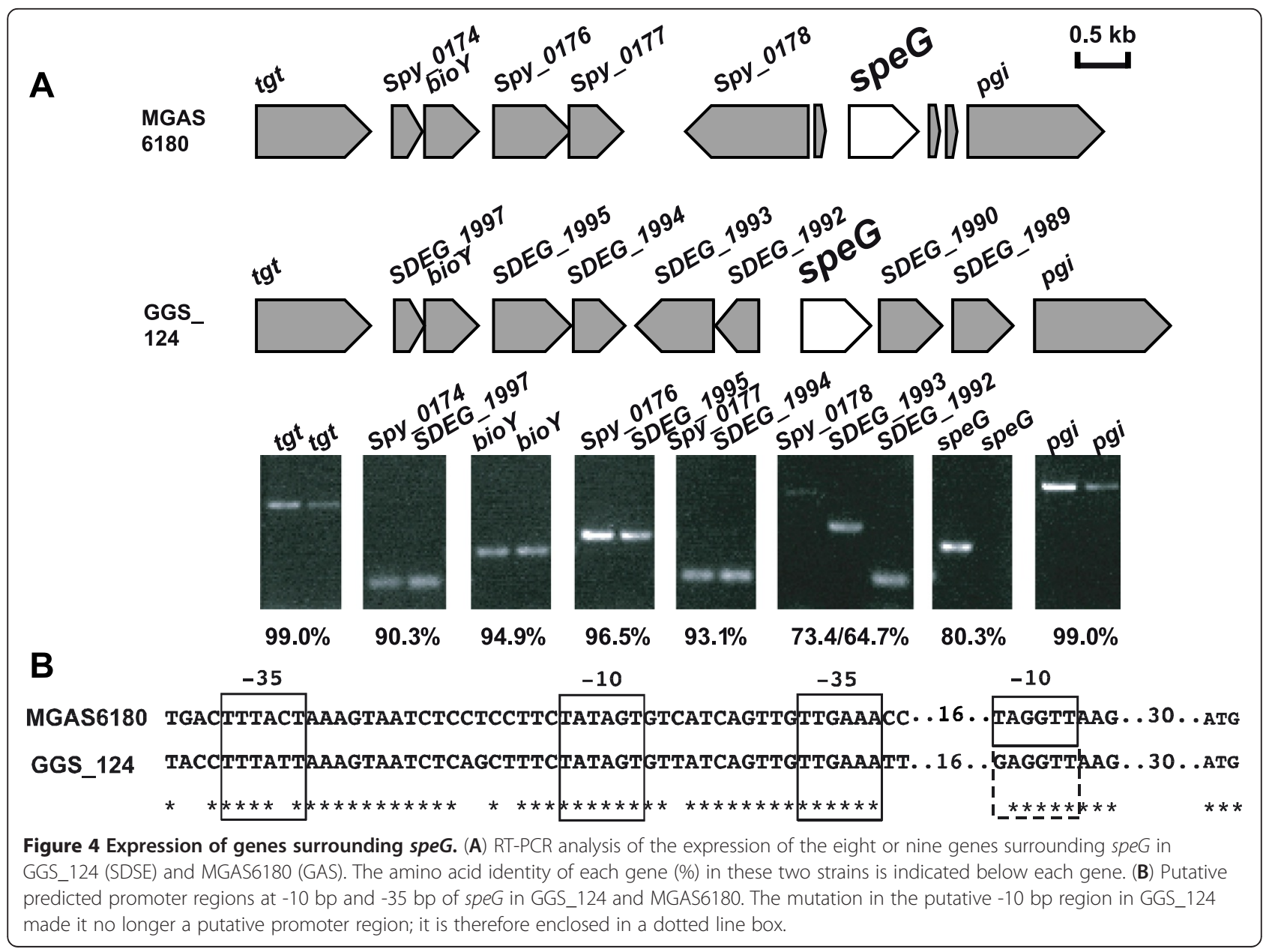


contrast, the mutations in the GGS_124 promoter candidates affected the promoter scores [28], making them no longer candidate promoters (data not shown). Our results were in good agreement with previous findings [25], in that none of the SDSE clinical strains expressed SpeG proteins, despite possessing intact speG genes.

\section{Analysis of the smez region}

We also analyzed the genome context surrounding smez, another chromosomally encoded SAg, in GAS and SDSE strains. We found that the synteny of approximately 20 $\mathrm{kb}$ regions containing smez (see details for MGAS10270 and NZ131, below) were highly conserved in all GAS strains sequenced (Figure 2). Of the 13 completely sequenced GAS strains, 11 harbored $s m e z$ genes, primarily at approximately $1.7 \mathrm{Mb}$. Although smez was present at the same site in SSI-1 as in the other GAS genomes, the former was functionally inactive due to a frame-shift mutation. In contrast, the MGAS10270 and NZ131 genomes did not contain smez fragments, even at other locations, despite their corresponding surrounding genome structures being highly conserved when compared with the other GAS strains. All GAS genomes contained highly similar $d p p$ operons $(d p p A, d p p B, d p p C, d p p D$, and $d p p E$ ) immediately downstream of smez, and all contained flaR and trpG, located upstream of smez coding sequences (Additional file 5).

Analysis of the GGS_124, RE378, and ATCC 12394 strains revealed that none contained fragments similar to the 702 bp smez coding sequence derived from the SF370 genome. We therefore searched for flaR and the $d p p$ operon, which were highly conserved in the smez flanking regions of GAS genomes (Figure 2). In these three SDSE genomes, flaR was located at about $0.2 \mathrm{Mb}$, whereas the $d p p$ operon was located at about $0.9 \mathrm{Mb}$, far from the position of flaR (Figure 2 and Additional file 5). Furthermore, synteny of the regions surrounding flaR and the $d p p$ operon was not well conserved in these three SDSE genomes, suggesting rearrangement of the genome context. The flaR gene and the $d p p$ operon show high similarities in GAS and SDSE (Additional file 5), with concomitant sequences observed only in GAS and SDSE but not in other streptococci (data not shown).

We next plotted cumulative TA-skew diagrams of the three sequenced SDSE chromosomes (GGS_124, RE378, and ATCC 12394). Use of a similar method on 12 sequenced GAS genomes showed that all cumulative TAskew curves of GAS genomes displayed a V-shape, interrupted by segments of steeper slopes, called steep-slope regions (SSRs) [29]. Diagram distortions including SSRs are thought to correspond to positions in which foreign genetic elements are integrated, including prophagerelated genes [30], horizontally acquired elements [31], and pathogenicity islands [29], and in which genome rearrangements occur [29]. The SSR was conserved among GAS strains, with smez at the border of the SSR, suggesting that this region is predisposed to be unstable [29].

Cumulative TA-skew curves of the three SDSE genomes formed a V-shape, similar to the GAS genomes (Figure 5B). GGS_124 contained four SSRs, whereas RE378 and ATCC 12394 contained three, some of which were located at similar positions (e.g. SSRs I and II, Figure 5B). These three SDSE genomes showed no evidence of massive genomic transversion or transition events (Figure 5A). Three of the four SSRs in the GGS_124 genome corresponded to prophages ФGGS_124.1, ФGGS_124.2, and ФGGS_124.4, with the fourth being a newly identified prophage-like element. In the RE378 genome, there was no correlation between the localization of two prophage-like elements and SSRs. Since SSR I and SSR II are conserved in all three genomes (Figure 5B), we further analyzed the genome contexts of SSR I and SSRII. The SSR I sequences from the three SDSE genomes varied in size and number of CDSs, with sizes ranging from 44 to $62 \mathrm{~kb}$. The number of CDSs included in SSR I also varied among the three SDSE genomes, but their core gene content was conserved, with high (>95\%) identity in the three genomes (Additional file 6). The Dpp operon, which is located in the region flanking smez in GAS strains (Figure 2), was contained in SSR I. This result is in good agreement with findings showing that the $d p p$ operon was located in a non-phagic SSR and conserved in all sequenced GAS genomes [29]. SSR II from the three SDSE genomes also varied in size, from 23 to $64 \mathrm{~kb}$, and number of CDSs. Although SSR II in the GGS_124 genome, at 1287230-1299098 bp, corresponded to the prophage region (Figure $5 \mathrm{~B}$ ), the core gene content in other nonphagic regions was conserved in all three genomes with high (> 95\%) similarities (Additional file 6). The presence of these two non-phagic SSRs suggests that another rearrangement event was involved in the formation of these SSRs. In one of the events in SSR I, smez was lost from the SDSE genome because the $d p p$ operon was a part of this SSR. These findings and the absence of smez from almost all SDSE strains [13] suggested that smez is a direct descendant of a common ancestor of streptococcal SAgs. Although this gene was conserved in GAS genomes, it was lost from SDSE due to a massive genome rearrangement that occurred after the speciation of SDSE and GAS.

\section{Identification of conserved enterotoxin like and staphylococcal superantigen like genes in all S. aureus genomes}

To determine the evolutionary pathway of staphylococcal SAgs, we analyzed all $14 \mathrm{~S}$. aureus genomes to 


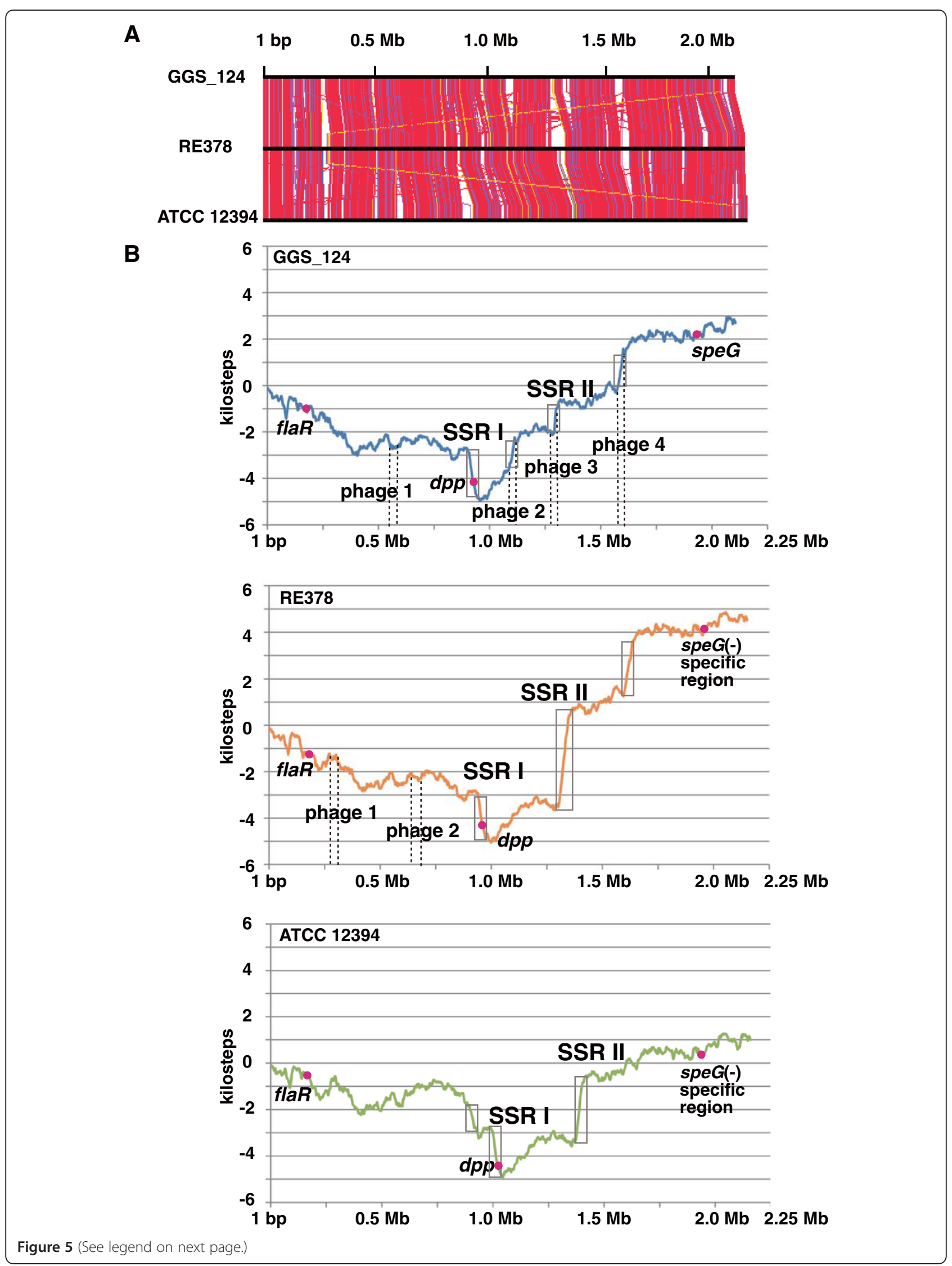


(See figure on previous page.)

Figure 5 Genome wide comparison among three SDSE genomes. (A) Genome rearrangement map among the three SDSE genomes. Each line joins two orthologues and the color of the lines represents the percentage of similarity between orthologous gene products (blue $\leq 40 \% \leq$ green $\leq 60 \% \leq$ yellow $\leq 70 \% \leq$ orange $\leq 80 \% \leq$ magenta $\leq 90 \% \leq$ red). (B) Cumulative TA-skews for the three SDSE genomes. Gray boxes represent SSRs. Each page region in the GGS_124 and RE378 genomes is indicated with broken lines. The X-axis represents nucleotide position.

determine whether their core chromosomes harbor orthologous staphylococcal enterotoxin-like (SEl) gene(s). We observed an SE-like gene (locus_tag SA1430) in S. aureus N315; this gene was designated the staphylococcal enterotoxin-like W (selW) gene according to guidelines [21] (Figure 6). We found no other candidate orthologous SE gene in these core genomes (data not shown). Surprisingly, SEIW has not yet been functionally analyzed, despite extensive study of staphylococcal SAgs.
This may be due, at least in part, to selW being annotated as SEA in the S. aureus genomes. We found, however, that the amino acid sequence of SEIW is only $36 \%$ identical to that of SEA, although phylogenetic analysis indicated that SEA is gene most similar to SEIW.

We found that the selWs are located at about 1.6 to $1.7 \mathrm{Mb}$ in the $S$. aureus genome, proximal to the $p f s$ gene encoding 5'-methylthioadenosine nucleosidase/ S-adenosylhomocysteine nucleosidase. Synteny of the selW

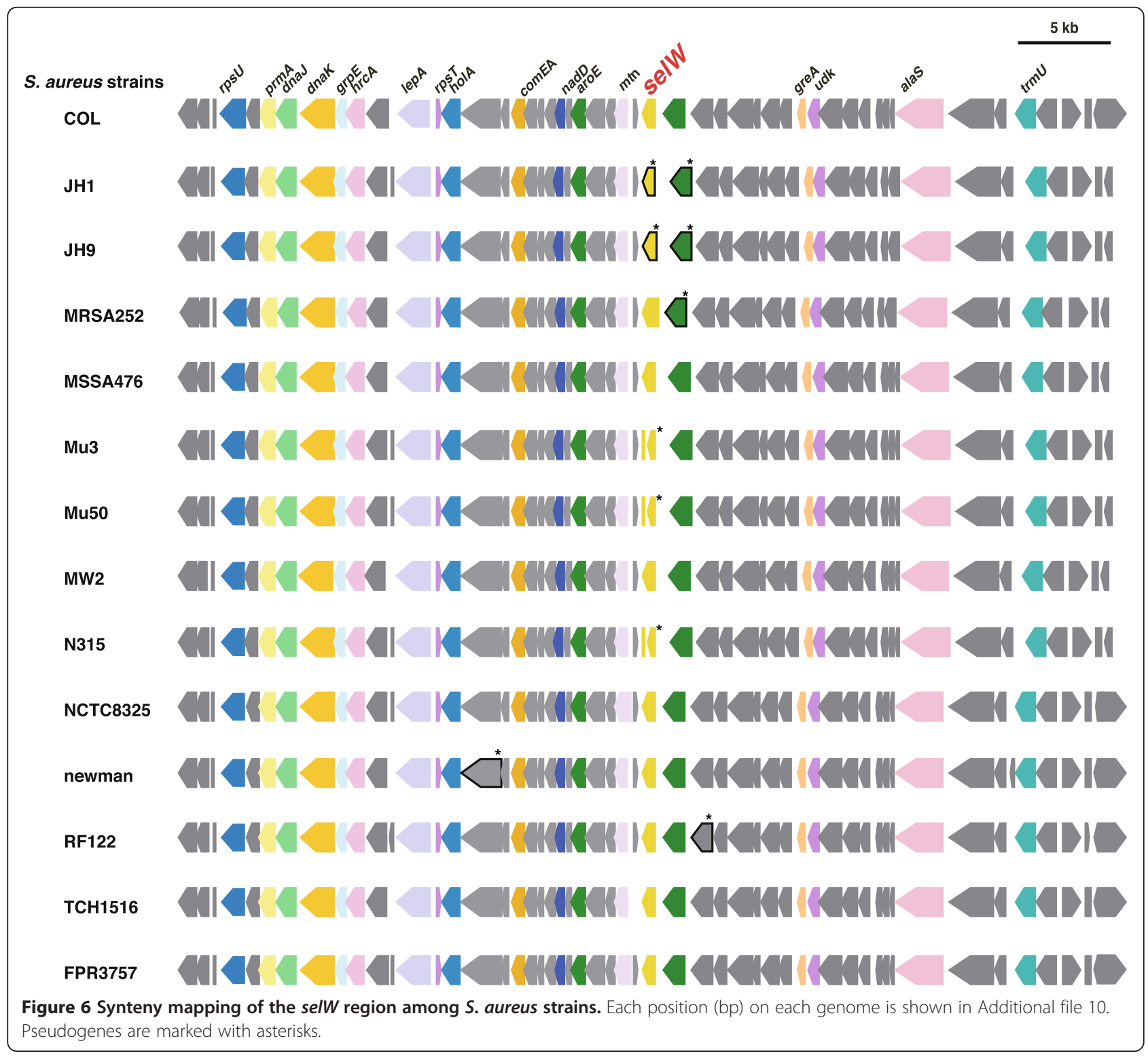


locus, including the proximal $50 \mathrm{~kb}$ regions, was found to be conserved in all $14 \mathrm{~S}$. aureus genomes available on the database (Figure 6). In contrast to other staphylococcal SAgs described to date, we detected no factor related to mobile genetic elements in these $50 \mathrm{~kb}$ regions. These findings strongly suggest that selW is a direct descendant of an ancestral staphylococcal SAg. To analyze whether selW has been subjected to positive selection, we performed window analysis for selW. Since no orthologous candidate genes were detected in other staphylococci or related bacteria, we performed our analysis using two selW genes from two different staphylococcus genomes. We found that several peaks had dN/dS ratios $>1 \quad$ (Figure 7), although these genes were derived from the same species. The ratios were especially high in the 3 ' region of selW. These results suggested that selW has been subjected to positive selection.

Analysis of the $14 \mathrm{~S}$. aureus genomes revealed three highly conserved ssl-like gene(s) in the core chromosome (ssl-12, ssl-13 and ssl-14; locus_tags SA1009, 1010, and 1011, respectively, in S. aureus N315) (Figure 8). Each of these genes showed approximately 30\% amino acid identity to ssl gene products. No other candidate ssl-like genes were present in any of the $S$. aureus genomes (data not shown). These genes have often been regarded as exotoxin- or superantigen-like genes, but they have not yet been functionally analyzed. The product of the SA1011 gene (S. aureus N315 ssl-14) had a C-terminal $\beta$-grasp domain (Pfam02876), which is a structural signature of SAg, whereas none had an N-terminal oligosaccharide-binding domain. The three genes are located at positions 1.1 to $1.3 \mathrm{Mb}$ of the S. aureus genome, upstream to the ornithine carbamoyltransferase $(\operatorname{arcB})$ and downstream to the alpha-hemolysin $(h l a)$ gene. In some strains such as JH1 and MRS252, hla is replaced by transposons, but synteny of the

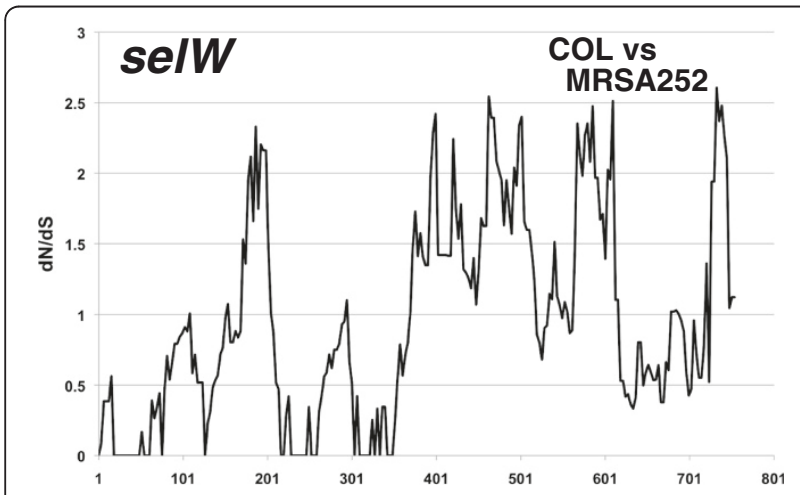

Figure 7 Window analysis calculating $d N / d S$ of selW. $d N / d S$ was calculated as the ratio of nonsynonymous (dN) to synonymous (dS) substitution rates of gene pairs in selW from two MRSA genomes. The figure shows only the comparison of COL and MRSA252. The $\mathrm{X}$-axis represents nucleotide position. upstream region remains highly conserved among these strains (Figure 8). Well-described SSLs are usually located in staphylococcal PIs, tandem structures consisting of three to ten SSL genes $[16,18,32]$. Since these ssl-like genes constitute a cluster of three homologous genes, their tandem structure in the PI is likely derived from their replication. To determine whether these ssl-like genes had been subjected to positive selection, we performed window analysis for ssl, using the three ssl-like genes, ssl-12, ssl-13 and ssl-14, located in the core chromosome. For comparison, we used Sca_0905 (Sc-set) derived from S. carnosus subsp. carnosus TM300, because its product showed significant similarities with products of the $s s l$-like cluster [33]. For each $s s l$-like gene, we observed several $\mathrm{dN} / \mathrm{dS}$ ratios greater than one (Figure 9). Although the three genes had slightly different patterns, high ratios were especially present in the middle and 3' regions of ssl. These findings suggest that positive selection has operated on these regions to create variations in staphylococcus SAgs and strongly suggest that these ssl-like genes are direct descendants of an ancestral staphylococcal SSL (SET).

$S$. aureus also harbors many types of SAgs, such as TSST-1, SEs, and SSLs. We identified a relatively unknown staphylococcal SAg, selW, and an ssl gene cluster, both of which are conserved in all $S$. aureus genomes examined to date. Moreover, we found that each of these genes was located in the same chromosomal region of the $S$. aureus genomes, not within any mobile elements. The highly syntenic conservation of selW and the ssl gene cluster among $S$. aureus genomes and their similarity to SEs and SSLs, respectively, suggest that they are likely the direct descendants of common ancestral SEs and SSLs, respectively.

\section{Evolutionary analysis of streptococcal and staphylococcal SAgs and SSL}

To determine the entire evolutionary pathway of streptococcal and staphylococcal SAgs and SSL, we constructed an evolutionary tree based on their nucleotide and amino acid sequences, including the newly identified SEIW and SSL-like cluster (Figure 10), using a Bayesian Markov chain Monte Carlo (MCMC) method. This approach, based on comparisons of the posterior probability of phylogenetic trees, allowed us to trace the evolutionary pathway of SAg in chronological order. In the resulting phylogenetic tree, streptococcal and staphylococcal SAgs and SSL could be divided into three groups, with clades I and II consisting of streptococcal SAgs and staphylococcal SSLs, respectively, and clade III consisting of both staphylococcal SEs and streptococcal SAgs (Figure 10). We found that each clade included at least one direct descendant in the core chromosome (e.g. SpeG and SMEZ for clade I, SSL-cluster and Sc-Set 


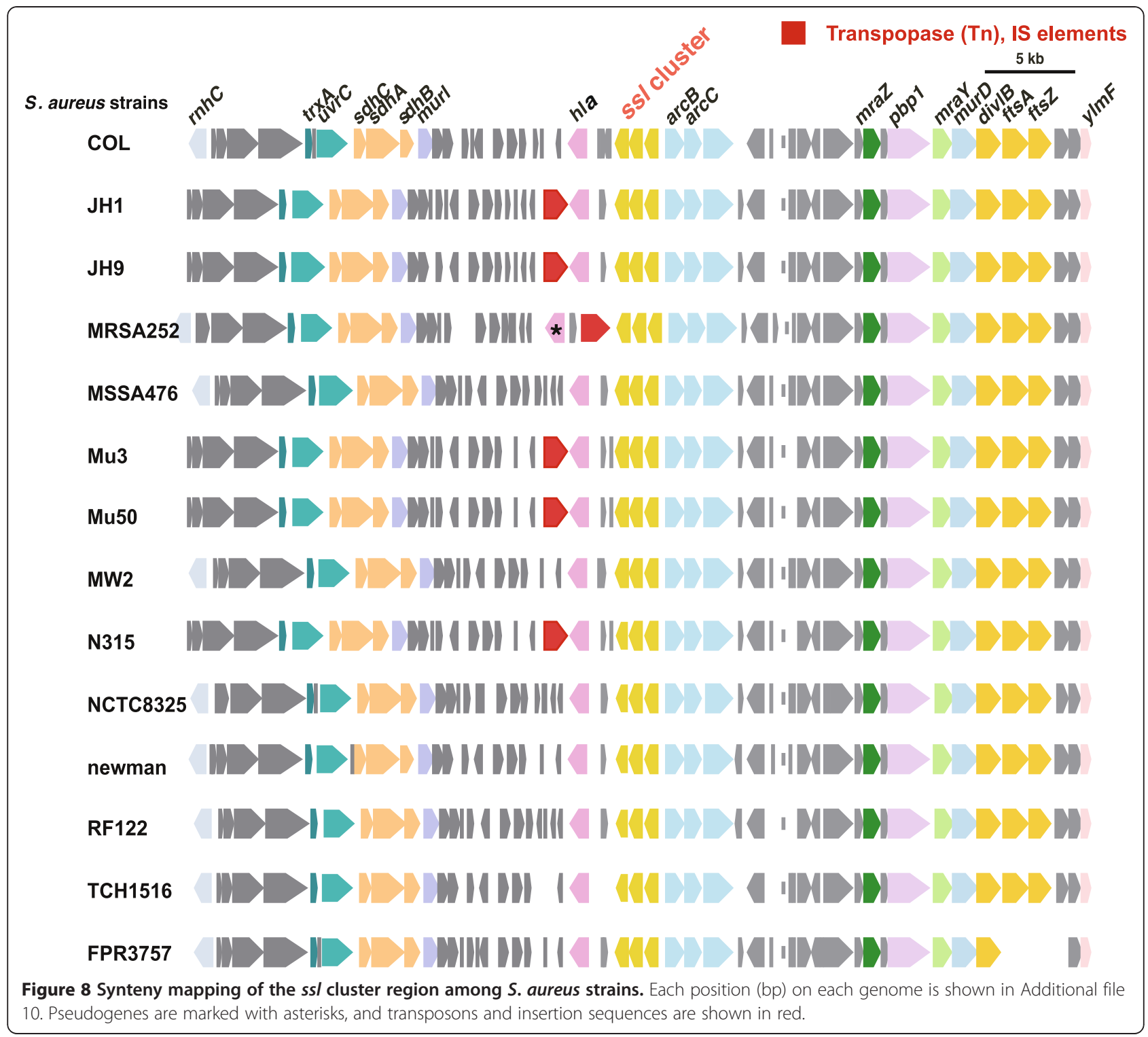

for clade II and SA-SEIW for clade III). A phylogenetic tree based on amino acid sequences was similar to that of the tree based on nucleotide sequences (Additional file 7). Because these trees were obtained by posterior probability analysis, it is highly likely that the SAgs in each clade evolved from their ancestors, which have been conserved in the core genome; i.e., SpeG and SMEZ in clade I, SSL in clade II and SEIW in clade III. Some streptococcal SAgs in clade III, including SpeA, SpeI, and SSA were more closely related to staphylococcal SAgs, including SEA, and SEB than to other streptococcal SAgs. Furthermore, although SEIW is located in the core chromosome (Figure 6), all of the streptococcal SAgs belonging to clade III are located in their prophages. These results suggest that some ancestral genes of streptococcal SAgs descended from staphylococcal
selW and that ancestral genes of selW were transferred from staphylococcal to streptococcal genomes.

The physiological activities and three-dimensional structures of SAgs are quite similar in streptococci and staphylococci. Although many studies have focused on staphylococcal SAgs in mobile elements, little is known about staphylococcal SAg-related gene(s) located on the core chromosome. To analyze the relationship among SAgs, we employed the Bayesian MCMC method. Although the phylogenetic tree we obtained was similar to that observed previously report [23], the method we used makes possible the determination of the temporal evolution of SAgs. Evolutionary analysis of the streptococcal and staphylococcal SAgs, and their related products, SSLs, showed that those molecules could be divided into three clades, each of which contains at least 


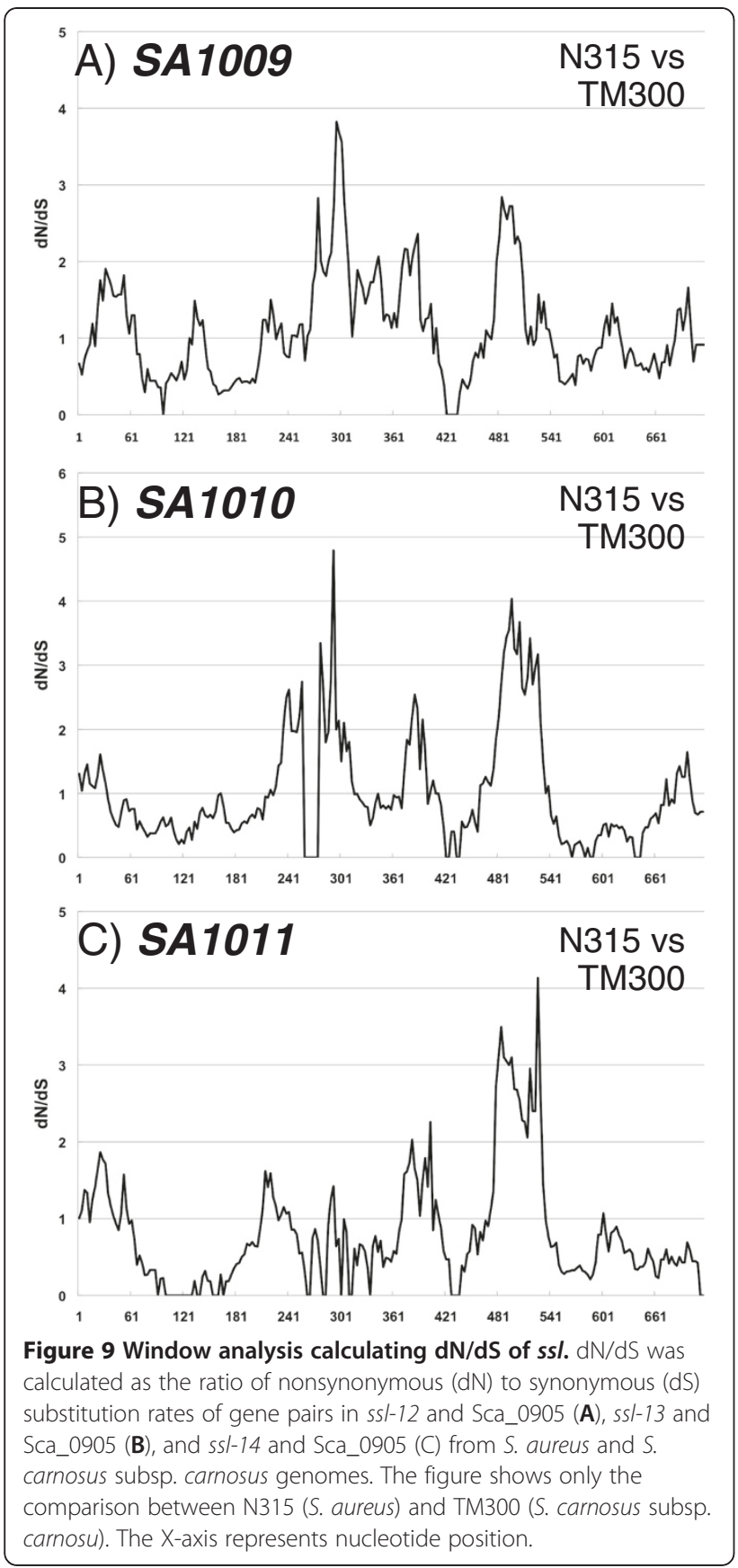

one direct descendant of an ancestor. SAgs of clades I and III consist of streptococcal and staphylococcal SAgs, respectively. In contrast, clade III is a mixture of streptococcal and staphylococcal SAgs, containing only SELW of $S$. aureus.

Based on these findings, we propose a model multi-step pathway for the evolution of SAgs (Figure 11). In step 1, the ancestors of streptococcal SAgs, SEs, and SSLs were acquired by ancestral bacteria. Detailed analyses of speG, smez, and selW were unable to determine additional ancestral forms, whereas the products of the ssl-like cluster were significantly similar to a product of Sca_0905 (Scset) derived from S. carnosus subsp. carnosus TM300 [33]. Although Sca_0905 itself is not conserved in S. aureus genomes, we found that the surrounding $50 \mathrm{~kb}$ regions were highly conserved in $S$. carnosus and $S$. aureus (Additional file 8). Thus, the ssl-like cluster may have arisen by multiplication of the ancestor of the Sca_0905 gene present in ancestral Staphylococcus. This hypothesis may be clarified when more genome sequences of $S$. carnosus become available. In step 2 of the evolutionary pathway, ancestral smez was likely deleted from the ancestral SDSE during a massive genome rearrangement driven by the SSR [29], whereas the ancestral speG survived in ancestral GAS and SDSE. Since speG is a dormant gene, it was likely replaced by a $20 \mathrm{~kb}$ fragment in ancestral SDSE strains soon after the speciation of GAS and SDSE. In step 3, the ancestral SAgs and SSLs were incorporated into mobile genetic elements, most likely phages, by chance, and transferred among bacterial strains. Phages derived from GAS can infect other species of Streptococcus [34-36]. A recombination-based model has been proposed for streptococcal toxins, including SAg dissemination among prophages [37]. This type of recombination event may drive molecular diversity. Bacteriophages in S. aureus have wide host ranges and potent lytic capability, and some carry staphylococcal SAgs such as SEC and TSST-1 [38]. It is highly likely that SEs and SSLs were duplicated during the transfer among bacteria. In step 4 of the evolutionary pathway, horizontal gene transfer occurred across species barriers. SaPI1 containing TSST-1, one of the most frequently observed SAgs in S. aureus, was shown to have been transferred to an evolutionarily distant species, Listeria monocytogenes, by staphylococcal phages [39]. Since no SAgs in clade I were closely similar to staphylococcal SAgs, horizontal transfer of SAgs likely occurred exclusively from Staphylococcus to Streptococcus.

\section{Conclusion}

Streptococcal SAgs are one of the important virulence factors involved in life-threatening diseases such as streptococcal toxic shock syndrome (STSS) and scarlet fever. At present, a total of 11 SAgs have been identified by GAS genome sequencing, with most GAS isolates possessing several SAg genes in their genomes. Although the diversity of SAgs is thought to arise during horizontal transfer, their evolutionary pathway has not been determined. To better understand SAg evolution, we sequenced the entire genome of SDSE, the closest relative of GAS, which harbors speG as its only SAg gene. Genome-wide comparisons of GAS and SDSE provided evidence that speG is the direct descendant of a common ancestor of the streptococcal SAg. Furthermore, we also detected previously undescribed inter-species horizontal SAg gene transfer events among three pathogens, 


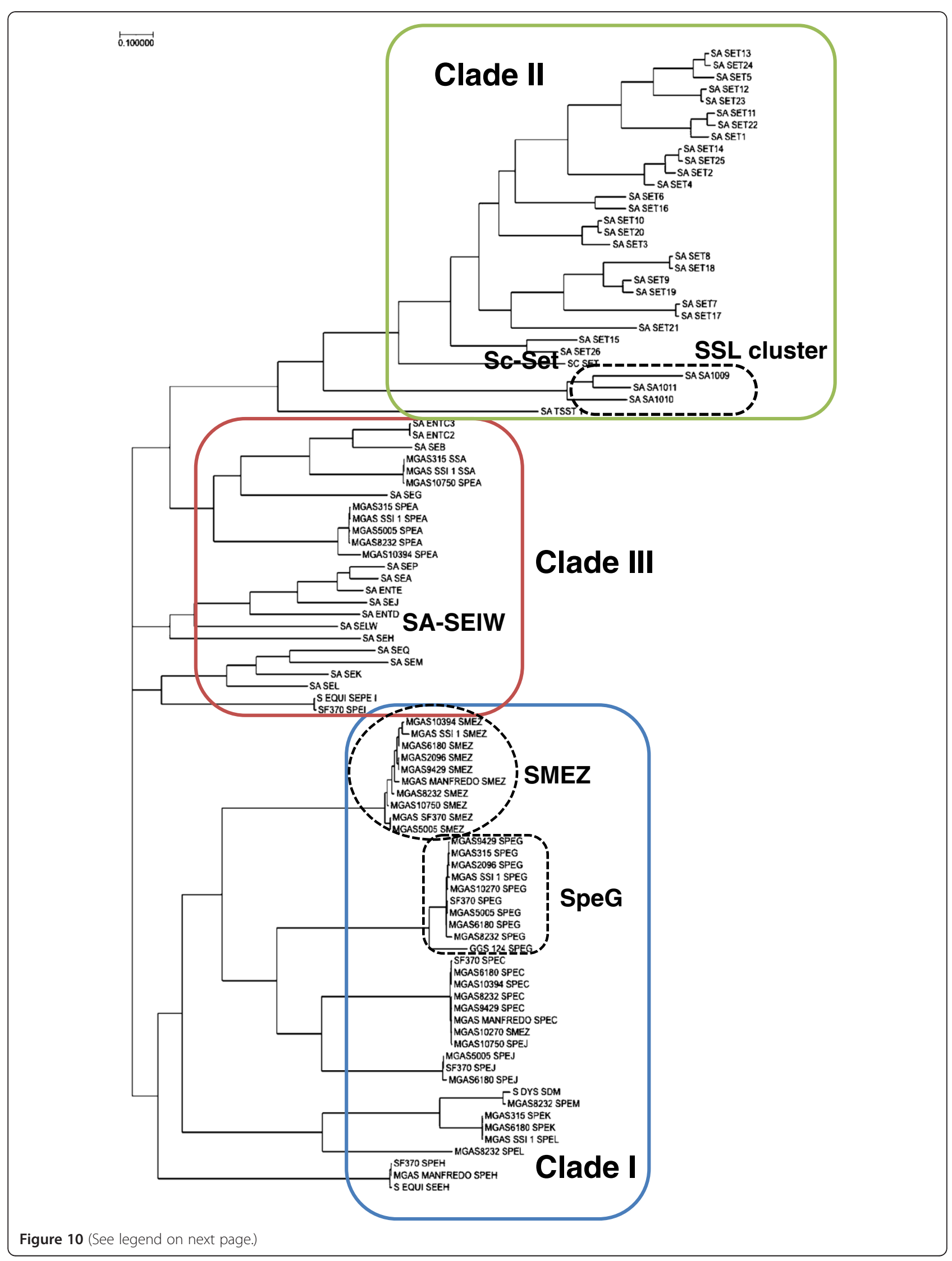


(See figure on previous page.)

Figure 10 Phylogenetic tree of streptococcal and staphylococcal SAgs and SSL nucleotide sequences. The phylogenetic tree was constructed using the Bayesian MCMC method, with 100,000 generations. The resultant potential scale reduction factor was 1.078. Essentially the same result was obtained by changing the number of generations and by using the amino acid evolution model (data not shown). The nucleotide sequences used for alignment are shown in Additional file 9. The resulting phylogenetic tree was composed of three clades, with clade I including only streptococcal SAgs, clade II including only staphylococcal SSLs, and clade III including SAgs from both species. Orthologous gene products, including SpeG and SMEZ in clade I, SSL-like proteins in clade II and SEIW in clade III, are emphasized.

S. pyogenes, S. dysgalactiae subsp. equisimilis and $S$. aureus. This study is the first time to describe the origin and evolution of SAgs in pathogenic streptococci and staphylococci. These findings suggest that horizontal gene transfer is a more ubiquitous genetic exchange system than previously known, and that it sometimes crosses interspecies barriers.

\section{Methods}

\section{Bacterial strains and media}

All S. dysgalactiae subsp. equisimilis (SDSE) strains used in this study were isolated from patients with invasive infections in different hospitals throughout Japan (Additional file 1). Each SDSE isolate was cultured in 5\% sheep blood agar or Brain Heart Infusion medium at $37^{\circ} \mathrm{C}$ under $5 \% \mathrm{CO}_{2}$ as described [8].

\section{Preparation of genomic DNA and sequencing}

Streptococci were lysed as described [8], and genomic DNA was purified using Wizard ${ }^{\circ}$ Genomic DNA Purification Kits (Promega).

PCR reactions were performed in volumes of $50 \mu \mathrm{l}$ containing TaKaRa ExTaq DNA polymerase (TaKaRa), with amplification on a GeneAmp PCR System 9700 (Applied Biosystems). Primer sets for direct sequencing were based on GGS_124 and RE378 genome sequence data, with each set designed to amplify $5 \mathrm{kbp}$ PCR products with 500 bp overlapping regions. The PCR primer set for the speG-specific region has been described [25] (see also Additional file 3). PCR products were electrophoresed on 1.0\% agarose gels and purified using QIAquick PCR Purification kits (QIAGEN). All DNA fragments were sequenced on an ABI3100 DNA sequencer with a redundancy of 4 .

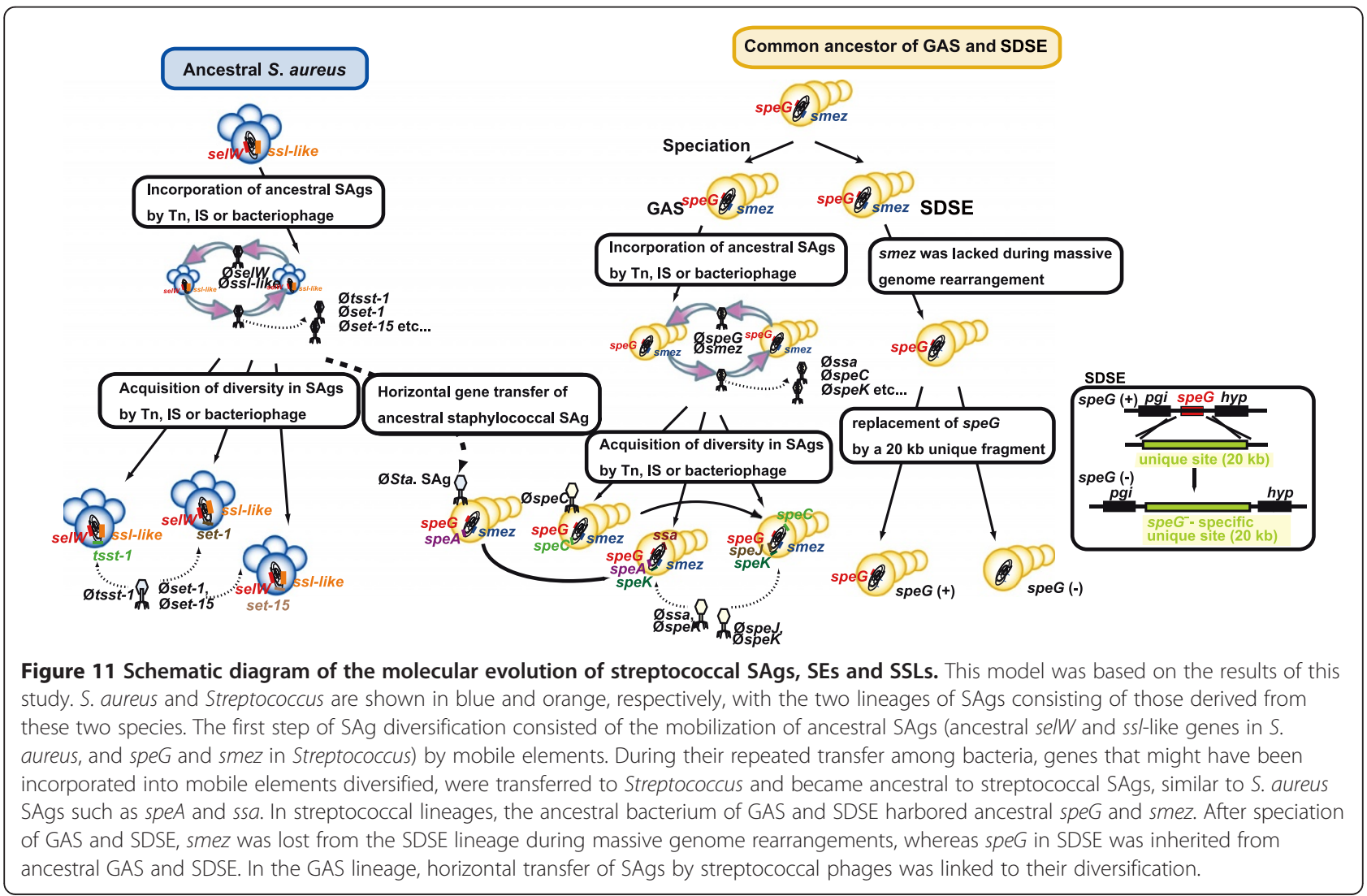




\section{RNA preparation}

All isolates were grown overnight in $10 \mathrm{ml}$ of Brain Heart Infusion medium at $37^{\circ} \mathrm{C}$ under $5 \% \mathrm{CO} 2$ in $15 \mathrm{ml}$ conical tubes. The cells were harvested, total RNA was purified using RNeasy Mini Kits (QIAGEN), and RNA concentrations were measured using a NanoDrop $^{\text {TM }} 1000$ spectrophotometer (Thermo Scientific).

\section{RT-PCR analysis of target genes}

Total RNA was reversed transcribed into cDNA using Superscript III reverse transcriptase kits (Invitrogen) and oligo dT primers. PCR amplifications were performed using the primer sequences in Additional file 3. The PCR products were electrophoresed on 1.0\% agarose gels and detected by UV-fluorescence after ethidium bromide staining.

\section{Bioinformatics and evolutionary analyses}

Homology searches and IS searches were performed using BLAST (http://blast.ncbi.nlm.nih.gov/Blast.cgi) and IS finder (http://www-is.biotoul.fr/is.html), respectively. Cumulative TA skew analysis was performed using GenSkew (http://genskew.csb.univie.ac.at/). Both window size and stepsize sequence length were set at $1000 \mathrm{bp}$. Rates of evolution were estimated by a Window Analysis of $\mathrm{dN}$ and $\mathrm{dS}$, using the online interface of WINA 0.34 [40], in a sliding window size of $60 \mathrm{bp}$ (20 codons) at $3 \mathrm{bp}$ intervals. A phylogenetic tree was constructed with CLUSTALW (http://clustalw.ddbj.nig.ac. jp/top-j.html), MrBayes 3.1.2 (http://mrbayes.csit.fsu.edu/ index.php) and TreeView X (http://darwin.zoology.gla.ac. $\mathrm{uk} / \sim \mathrm{rpage} /$ treeviewx/index.html) software. Sequences were manually corrected using GENETYX-Mac (GENETYX Co.) and gene analysis was performed by in silico molecular cloning (in silico Biology Co.).

\section{Nucleotide and amino acid sequence accession number}

The DNA sequences of the region surrounding speG $(10-60 \mathrm{~kb})$ in each strain have been deposited in the DDBJ under the accession numbers listed in Additional file 1. Accession numbers for SAgs used in this study are listed in Additional file 9.

\section{Additional files}

Additional file 1: Streptococcus isolates used in this study.

Additional file 2: Homology of $S$. dysgalactiae subsp. equisimilis GGS_124 with other bacteria at the genome level.

Additional file 3: List of oligonucleotide primers used in this study.

Additional file 4: Features of speG-negative strain specific regions.

Additional file 5: Summary of flaR and the $d p p$ operon in each Streptococci strain.

Additional file 6: List of genes present in SSRI and SSR II.
Additional file 7: Phylogenetic tree of streptococcal and staphylococcal SAgs and SSL amino acid sequences. The phylogenetic tree was constructed using the Bayesian MCMC method, with 100,000 generations and a mixed amino acid evolution model. The resultant potential scale reduction factor was 1.078. Essentially the same result was obtained by changing the number of generations and using the amino acid evolution model (data not shown). The nucleotide sequences used for alignment are shown in Additional file 9. The resulting phylogenetic tree was composed of three clades, with clade I including only streptococcal SAgs, clade II including only staphylococcal SSLS, and clade III including SAgs from both species. Orthologous gene products, including SpeG and SMEZ in clade I, SSL-like proteins in clade II and SEIW in clade III, are emphasized. Yersinia SAgs (YPMA, YPMB, and YPMC) were also included as an outgroup.

Additional file 8: Synteny mapping of the set-containing regions of Staphylococcus carnosus TM300 and S. aureus strains. Each position (bp) on each genome is shown in Additional file 10.

Additional file 9: List of Accession numbers for SAgs used in this study.

Additional file 10: List of positions (bp) on each genome.

Competing interests

The authors declare no competing financial interests.

\section{Authors' contributions}

$\mathrm{KO}$ performed the direct sequencing analyses and RT-PCR assays. KO and TM-A performed the bioinformatic analyses. KO, YS, SY-M and TM-A performed GGS_124 and RE378 genome sequence analyses. SY-M and KU provided isolates for the analyses. KU, TK and TM-A. supervised the project. JY and TK coordinated the project. KO and TM-A prepared the manuscript. All authors read and approved the final manuscript.

\section{Acknowledgements}

The authors thank Dr. Omoe at Iwate University for valuable discussions about the evolution of SEs. This work was partly supported by a grant for Research on Emerging and Reemerging Infectious Diseases (H22 Shinkouh013). T. M. A was supported by JSPS KAKENHI Grant Number 21590503 and 24390109.

\section{Author details}

'Department of Infectious Diseases, National Center for Global Health and Medicine, 1-21-1, Shinjuku-ku, Tokyo 162-8655, Japan. ${ }^{2}$ Graduate School of Infection Control Science, Kitasato University, 5-9-1, Shirokane, Minato-ku, Tokyo108-8641, Japan. ${ }^{3}$ Department of Microbiology and Immunology, Tokyo Women's Medical University, 8-1 Kawada-cho, Shinjuku-ku, Tokyo 162-8666, Japan. ${ }^{4}$ Department of Animal and Food Hygiene, Obihiro University of Agriculture and Veterinary Medicine, Inada-cho, Obihiro, Hokkaido 080-8555, Japan. ${ }^{5}$ Present affiliation: College of Pharmacy, Nihon University, Narashinodai, Funabashi, Chiba 274-8555, Japan.

Received: 3 April 2012 Accepted: 30 June 2012

Published: 17 August 2012

\section{References}

1. Alouf JE, Müller $\mathrm{H}$ : What are superantigens? In The comprehensive sourcebook of bacterial protein toxins. 3rd edition. Edited by Alouf JE, Popoff MR. New York: Academic Press; 2006:821-829.

2. Fraser JD, Proft T: The bacterial superantigen and superantigen-like proteins. Immunol Rev 2008, 225:226-243.

3. Brosnahan AJ, Schlievert PM: Gram-positive bacterial superantigen outside-in signaling causes toxic shock syndrome. FEBS J 2011, 278(23):4649-4667.

4. Shimomura Y, Okumura K, Murayama SY, Yagi J, Ubukata K, Kirikae T, Miyoshi-Akiyama T: Complete genome sequencing and analysis of a Lancefield group G Streptococcus dysgalactiae subsp. equisimilis strain causing streptococcal toxic shock syndrome (STSS). BMC Genomics 2011, 12(1):17. 
5. McDonald M, Towers RJ, Andrews RM, Carapetis JR, Currie BJ: Epidemiology of Streptococcus dysgalactiae subsp. equisimilis in tropical communities, Northern Australia. Emerg Infect Dis 2007, 13(11):1694-1700.

6. Tanaka D, Isobe J, Watahiki M, Nagai Y, Katsukawa C, Kawahara R, Endoh M, Okuno R, Kumagai N, Matsumoto M, Working Group for Group A Streptococci in Japan, et al: Genetic features of clinical isolates of Streptococcus dysgalactiae subsp. equisimilis possessing Lancefield's group A antigen. J Clin Microbiol 2008, 46(4):1526-1529.

7. Broyles LN, Van Beneden C, Beall B, Facklam R, Shewmaker PL, Malpiedi P, Daily P, Reingold A, Farley MM: Population-based study of invasive disease due to beta-hemolytic streptococci of groups other than $A$ and B. Clin Infect Dis 2009, 48(6):706-712.

8. Miyoshi-Akiyama T, Zhao J, Kato H, Kikuchi K, Totsuka K, Kataoka Y, Katsumi M, Uchiyama T: Streptococcus dysgalactiae-derived mitogen (SDM), a novel bacterial superantigen: characterization of its biological activity and predicted tertiary structure. Mol Microbiol 2003, 47(6):1589-1599.

9. Proft T, Webb PD, Handley V, Fraser JD: Two novel superantigens found in both group A and group C Streptococcus. Infect Immun 2003, 71(3):1361-1369.

10. Thomas P, Webb PD, Handley V, Fraser JD: Identification \& characterisation of the two novel streptococcal pyrogenic exotoxins SPE-L \& SPE-M. Indian J Med Res 2004, 119(Suppl):37-43.

11. Alber J, El-Sayed A, Estoepangestie S, Lammler C, Zschock M: Dissemination of the superantigen encoding genes seeL, seeM, szeL and szeM in Streptococcus equi subsp. equi and Streptococcus equi subsp. zooepidemicus. Vet Microbiol 2005, 109(1-2):135-141.

12. Igwe El, Shewmaker PL, Facklam RR, Farley MM, van Beneden C, Beall B: Identification of superantigen genes speM, ssa, and smeZ in invasive strains of beta-hemolytic group C and G streptococci recovered from humans. FEMS Microbiol Lett 2003, 229(2):259-264.

13. Davies MR, McMillan DJ, Beiko RG, Barroso V, Geffers R, Sriprakash KS, Chhatwal GS: Virulence profiling of Streptococcus dysgalactiae subspecies equisimilis isolated from infected humans reveals 2 distinct genetic lineages that do not segregate with their phenotypes or propensity to cause diseases. Clin Infect Dis 2007, 44(11):1442-1454.

14. Hashikawa S, linuma Y, Furushita M, Ohkura T, Nada T, Torii K, Hasegawa T, Ohta M: Characterization of group $C$ and $\mathrm{G}$ streptococcal strains that cause streptococcal toxic shock syndrome. J Clin Microbiol 2004, 42(1):186-192.

15. Brandt CM, Schweizer KG, Holland R, Lutticken R, Freyaldenhoven BS: Lack of mitogenic activity of speG- and spe $^{\text {dys }}$-positive Streptococcus dysgalactiae subspecies equisimilis isolates from patients with invasive infections. Int J Med Microbiol 2005, 295(8):539-546.

16. Baba T, Takeuchi F, Kuroda M, Yuzawa H, Aoki K, Oguchi A, Nagai Y, Iwama N, Asano K, Naimi T, et al: Genome and virulence determinants of high virulence community-acquired MRSA. Lancet 2002, 359(9320):1819-1827.

17. Ruzin A, Lindsay J, Novick RP: Molecular genetics of SaPI1-a mobile pathogenicity island in Staphylococcus aureus. Mol Microbiol 2001, 41 (2):365-377.

18. Williams RJ, Ward JM, Henderson B, Poole S, O'Hara BP, Wilson M, Nair SP: Identification of a novel gene cluster encoding staphylococcal exotoxinlike proteins: characterization of the prototypic gene and its protein product, SET1. Infect Immun 2000, 68(8):4407-4415.

19. Arcus VL, Proft T, Sigrell JA, Baker HM, Fraser JD, Baker EN: Conservation and variation in superantigen structure and activity highlighted by the three-dimensional structures of two new superantigens from Streptococcus pyogenes. J Mol Biol 2000, 299(1):157-168.

20. Mitchell DT, Levitt DG, Schlievert PM, Ohlendorf DH: Structural evidence for the evolution of pyrogenic toxin superantigens. J Mol Evol 2000, 51(6):520-531

21. Lina G, Bohach GA, Nair SP, Hiramatsu K, Jouvin-Marche E, Mariuzza R, International Nomenclature Committee for Staphylococcal Superantigens: Standard nomenclature for the superantigens expressed by Staphylococcus. J Infect Dis 2004, 189(12):2334-2336.

22. Kapur V, Reda KB, Li LL, Ho LJ, Rich RR, Musser JM: Characterization and distribution of insertion sequence IS1239 in Streptococcus pyogenes. Gene 1994, 150(1):135-140.

23. Sachse S, Seidel P, Gerlach D, Gunther E, Rodel J, Straube E, Schmidt KH: Superantigen-like gene(s) in human pathogenic Streptococcus dysgalactiae subsp. equisimilis: genomic localisation of the gene encoding streptococcal pyrogenic exotoxin G (speG ${ }^{\text {dys }}$ ). FEMS Immunol Med Microbiol 2002, 34(2):159-167.
24. Koonin EV, Makarova KS, Aravind L: Horizontal gene transfer in prokaryotes: quantification and classification. Annu Rev Microbiol 2001, 55:709-742.

25. Zhao J, Hayashi T, Saarinen S, Papageorgiou AC, Kato H, Imanishi K, Kirikae T, Abe R, Uchiyama T, Miyoshi-Akiyama T: Cloning, expression, and characterization of the superantigen streptococcal pyrogenic exotoxin $\mathrm{G}$ from Streptococcus dysgalactiae. Infect Immun 2007, 75(4):1721-1729.

26. Steer AC, Law I, Matatolu L, Beall BW, Carapetis JR: Global emm type distribution of group A streptococci: systematic review and implications for vaccine development. Lancet Infect Dis 2009, 9(10):611-616.

27. Suzuki H, Lefebure T, Hubisz MJ, Pavinski Bitar P, Lang P, Siepel A, Stanhope MJ: Comparative genomic analysis of the Streptococcus dysgalactiae species group: gene content, molecular adaptation, and promoter evolution. Genome Biol Evol 2011, 3:168-185.

28. Mulligan ME, Hawley DK, Entriken R, McClure WR: Escherichia coli promoter sequences predict in vitro RNA polymerase selectivity. Nucleic Acids Res 1984, 12(1 Pt 2):789-800.

29. Panchaud A, Guy L, Collyn F, Haenni M, Nakata M, Podbielski A, Moreillon P, Roten CA: M-protein and other intrinsic virulence factors of Streptococcus pyogenes are encoded on an ancient pathogenicity island. BMC Genomics 2009, 10:198.

30. Roten CA, Gamba P, Barblan UL, Karamata D: Comparative Genometrics (CG): a database dedicated to biometric comparisons of whole genomes. Nucleic Acids Res 2002, 30(1):142-144.

31. Grigoriev A: Analyzing genomes with cumulative skew diagrams. Nucleic Acids Res 1998, 26(10):2286-2290.

32. Highlander SK, Hulten KG, Qin X, Jiang H, Yerrapragada S, Mason EO Jr Shang Y, Williams TM, Fortunov RM, Liu Y, et al: Subtle genetic changes enhance virulence of methicillin resistant and sensitive Staphylococcus aureus. BMC Microbiol 2007, 7:99.

33. Rosenstein R, Nerz C, Biswas L, Resch A, Raddatz G, Schuster SC, Gotz F: Genome analysis of the meat starter culture bacterium Staphylococcus carnosus TM300. Appl Environ Microbiol 2009, 75(3):811-822.

34. Davies MR, McMillan DJ, Van Domselaar GH, Jones MK, Sriprakash KS: Phage 3396 from a Streptococcus dysgalactiae subsp. equisimilis pathovar may have its origins in Streptococcus pyogenes. J Bacterio/ 2007, 189(7):2646-2652.

35. Vojtek I, Pirzada ZA, Henriques-Normark B, Mastny M, Janapatla RP, Charpentier E: Lysogenic transfer of group A Streptococcus superantigen gene among Streptococci. J Infect Dis 2008, 197(2):225-234

36. Holden MT, Heather Z, Paillot R, Steward KF, Webb K, Ainslie F, Jourdan T, Bason NC, Holroyd NE, Mungall K, et al: Genomic evidence for the evolution of Streptococcus equi: host restriction, increased virulence, and genetic exchange with human pathogens. PLoS Pathog 2009, 5(3): e1000346.

37. Aziz RK, Edwards RA, Taylor WW, Low DE, McGeer A, Kotb M: Mosaic prophages with horizontally acquired genes account for the emergence and diversification of the globally disseminated M1T1 clone of Streptococcus pyogenes. J Bacteriol 2005, 187(10):3311-3318.

38. Synnott AJ, Kuang Y, Kurimoto M, Yamamichi K, Iwano H, Tanji Y: Isolation from sewage influent and characterization of novel Staphylococcus aureus bacteriophages with wide host ranges and potent lytic capabilities. Appl Environ Microbiol 2009, 75(13):4483-4490.

39. Chen J, Novick RP: Phage-mediated intergeneric transfer of toxin genes. Science 2009, 323(5910):139-141.

40. Endo T, Ikeo K, Gojobori T: Large-scale search for genes on which positive selection may operate. Mol Biol Evol 1996, 13(5):685-690.

doi:10.1186/1471-2164-13-404

Cite this article as: Okumura et al.: Evolutionary paths of streptococcal and staphylococcal superantigens. BMC Genomics 2012 13:404. 